\title{
Research on Knowledge-sharing Continuance Social Intention for Social Q\&A Community
}

\author{
Longyi Li ${ }^{1, a}$,Tao $\mathrm{Yu}^{2, b^{*}}$,Qiong Wang ${ }^{3, \mathrm{c}}$
}

${ }^{1}$ South China University of Technology,Guangzhou ,Guangdong,China

2South China University of Technology,Guangzhou ,Guangdong,China

${ }^{3}$ South China University of Technology, Guangzhou ,Guangdong,China

a198143297@qq.com,b191463900@qq.com,c8143955558@qq.com

Keywords: organizational commitment,self-efficacy, knowledge sharing

\begin{abstract}
Based on the organizational commitment theory and self-efficacy theory, this paper constructs a social Q\&A community user model for knowledge sharing behavior from four dimensions: Affective commitment, continuance commitment, normative commitment and knowledge self-efficacy. Moreover, the model and hypotheses are verified by the empirical study through collected sample data.
\end{abstract}

\section{社交问答社区用户持续知识共享意愿实证研究 \\ 李龙一 $1, \mathrm{a}$,余 涛 $2, \mathrm{~b}^{*}$, 王琼 $3, \mathrm{c}$ \\ 1 华南理工大学, 广州, 广东, 中国 \\ 2华南理工大学, 广州, 广东, 中国 \\ 3华南理工大学, 广州, 广东, 中国 \\ a198143297@qq.com, b191463900@qq.com, ${ }^{c 8143955558 @ q q . c o m}$}

关键词: 组织承诺, 自我效能, 知识共享

中文摘要: 本文以组织承诺理论和自我效能理论为基础, 从情感承诺、持续承诺、规范承诺 和知识自我效能四个维度出发, 构建了社交问答社区用户持续知识共享行为模型, 通过收集 的样本数据对模型和假设进行实证检验。

\section{1 引言}

虚拟社区作为一种新的交流方式和沟通媒介，为广大网民分享信息与知识提供了有效载 体和平台，基于虚拟社区的沟通模式被认为是推动知识共享的有效方法之一。本研究选取社 交问答社区作为研究背景, 从组织行为学和社会心理学的视角探讨并实证分析其对社区用户 持续知识共享行为意愿的作用机理, 以期进一步深化和拓展当前研究。

\section{2 文献综述}

\section{1 组织承诺理论}

美国社会学家 Becker 是研究组织承诺的第一位学者, 他指出个体希望能够从组织的投入 
中得到回报, 从而对组织产生承诺[1]。组织行为学学者 Buchanan 和 Porter 从心理学的角度指 出承诺主要表现为员工对组织的感情依赖, 以及个体对组织目标和价值观的一种感情依附 [2]。Mayer 和 Allen 于 1990 年提出的三维结构模型, 他们将组织承诺分为情感承诺、持续承 诺和规范承诺三个维度，分别表征组织成员对组织在情感、经济和规范上的承诺[3]。

\section{2 自我效能理论}

1977 年, 美国心理学学者 Bandura 首次提出自我效能的概念, 他认为, 个人在面对特殊 情景时, 对如何才能有效开展行动方案的判断和信念就是自我效能。自我效能是 “个体对自 身是否具有完成某一活动所具备的能力的判断和信念” 或 “个体在完成某一活动或任务时的 胜任感、自信、自尊等感受”, 即 “人们对自身完成某项任务或工作的能力所抱有的信念, 它 并不涉及技能本身，而是自己对利用所掌握的技能来完成工作的自信程度” [4]。

\section{3 研究假设与模型}

\section{1 研究假设}

$\mathrm{H} 1$ ：情感承诺正向影响社交问答平台的用户满意度。

$\mathrm{H} 2$ ：持续承诺正向影响社交问答平台的用户满意度。

H3：规范承诺正向影响社交问答平台的用户满意度。

$\mathrm{H} 4$ ：知识自我效能正向影响用户参与社交问答平台的满意度。

H5：知识自我效能正向影响用户参与社交问答平台的意愿。

H6: 用户满意度与其持续参与社交问答平台知识贡献意愿正相关。

根据以上假设, 本构建了如图 1 的研究模型

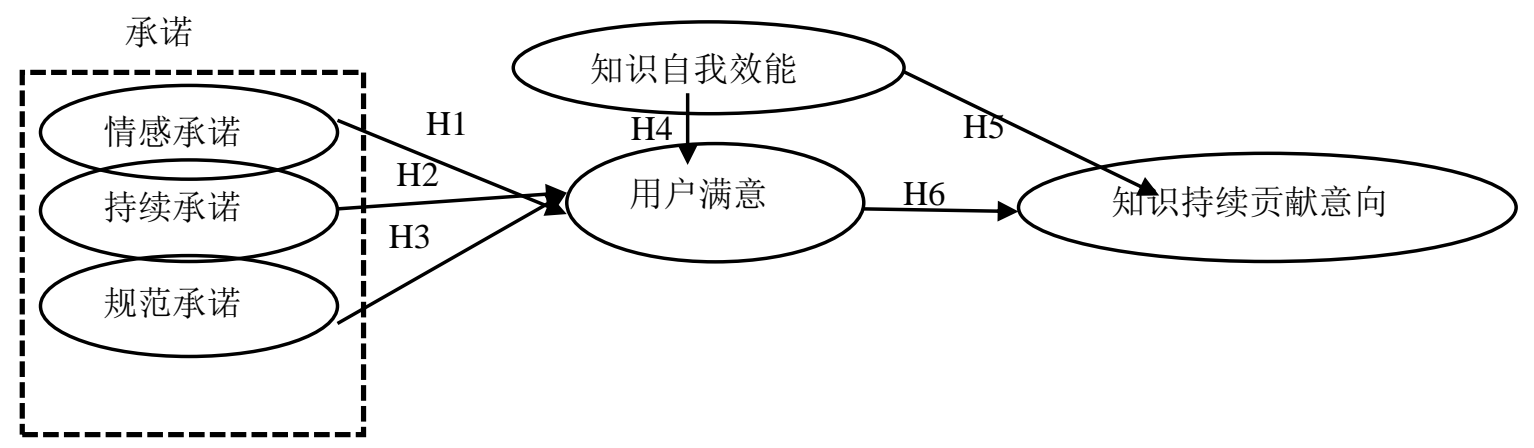

图 1 社交问答社区成员持续知识贡献意愿研究模型

\section{4 研究方法}

\section{1 量表设计}

设计问卷采用李克特 7 级量表，量表的题项主要来源于国内外相关文献，并结合社交问 答平台本身的特点进行调整。问卷初步设计好后, 在 50 位深度知乎用户中进行了预调研, 并 对 5 位资深知乎用户进行访谈，经过修改后得到最终量表。

\section{2 数据来源}

本研究采用了问卷调查的方法收集数据, 问卷通过问卷星平台发布。调查对象主要选取 国内最大的社交问答平台知乎的用户。任务有限期为一个月，共收集到问卷 508 份，剔除 191 份无效问卷后，最终得到有效问卷 278 份，问卷有效率达 $87.7 \%$ 。 


\section{5 数据处理}

\section{1 样本描述性统计}

样本男性略多于女性，男女比例分别为 59\%和 $41 \%$; 且各个年龄层都有覆盖，整体结构 偏年轻化，19-35 岁之间的受访者占到所有样本的 96.1\%, 这与使用知乎的主要用户群体基本 一致, 因此, 本研究受访者的年龄构成较具说服力。

\section{2 信度与效度}

李克特量表对信度通常用 Cronbach Alpha 值和组合信度来检验, 一般大于 0.7 则可认为测 量题项的设计是可靠的。同时组合信度值 (CR) 大于 0.7 则表明模型的内在质量良好。经检 验, 所有的 Cronbach Alpha 值均大于 0.7, 所有变量的组合信度值 (CR) 均大于 0.8 , 说明问 卷是可靠的, 且具有较好稳定性。收玫效度一般用 AVE (平均变异萃取量) 来评估, 一般要 求 AVE 大于 0.5.经检验发现所有变量的 AVE 均大于 0.5（见表 1)，说明问卷收敛效度良好。

表 1 测量信度和效度

\begin{tabular}{|c|c|c|c|c|c|}
\hline 潜变量 & 测量项 & 因子载荷 & $\mathrm{CR}$ & AVE & Alpha \\
\hline \multirow{3}{*}{$\begin{array}{c}\text { 情感承诺 } \\
(\mathrm{AC})\end{array}$} & $\mathrm{AC1}$ & 0.876 & \multirow{3}{*}{0.901} & \multirow{3}{*}{0.652} & \multirow{3}{*}{0.924} \\
\hline & AC2 & 0.792 & & & \\
\hline & AC3 & 0.801 & & & \\
\hline \multirow{3}{*}{$\begin{array}{c}\text { 持续承诺 } \\
\text { (CC) }\end{array}$} & $\mathrm{CC} 1$ & 0.806 & \multirow{3}{*}{0.897} & \multirow{3}{*}{0.685} & \multirow{3}{*}{0.914} \\
\hline & $\mathrm{CC} 2$ & 0.870 & & & \\
\hline & $\mathrm{CC} 3$ & 0.820 & & & \\
\hline \multirow{3}{*}{$\begin{array}{c}\text { 规范承诺 } \\
\text { （NC） }\end{array}$} & $\mathrm{NC1}$ & 0.902 & \multirow{3}{*}{0.934} & \multirow{3}{*}{0.779} & \multirow{3}{*}{0.918} \\
\hline & NC2 & 0.891 & & & \\
\hline & $\mathrm{NC3}$ & 0.872 & & & \\
\hline \multirow{3}{*}{$\begin{array}{l}\text { 知识自我效能 } \\
\text { (KSE) }\end{array}$} & KSE1 & 0.702 & \multirow{3}{*}{0.859} & \multirow{3}{*}{0.605} & \multirow{3}{*}{0.917} \\
\hline & KSE2 & 0.786 & & & \\
\hline & KSE3 & 0.803 & & & \\
\hline \multirow{3}{*}{$\begin{array}{l}\text { 用户满意度 } \\
\text { (US) }\end{array}$} & US1 & 0.834 & \multirow{3}{*}{0.871} & \multirow{3}{*}{0.629} & \multirow{3}{*}{0.950} \\
\hline & US2 & 0.824 & & & \\
\hline & US3 & 0.731 & & & \\
\hline \multirow{3}{*}{$\begin{array}{l}\text { 持续知识贡献意向 } \\
\text { (CKCI) }\end{array}$} & CKCI1 & 0.821 & \multirow{3}{*}{0.882} & \multirow{3}{*}{0.612} & \multirow{3}{*}{0.930} \\
\hline & CKCI2 & 0.734 & & & \\
\hline & CKCI3 & 0.783 & & & \\
\hline
\end{tabular}

本文模型的路径系数和假设检验结果如下。

表 2 拟合指数建议值及实际值

\begin{tabular}{lccccccc}
\hline 拟合指标 & $\mathrm{Chi}^{2} / \mathrm{df}$ & GFI & AGFI & CFI & NFI & TLI & RMSEA \\
\hline 推荐值 & $<3$ & $>0.90$ & $>0.80$ & $>0.90$ & $>0.90$ & $>0.90$ & $<0.08$ \\
实际值 & 1.826 & 0.912 & 0.883 & 0.953 & 0.903 & 0.944 & 0.053 \\
\hline
\end{tabular}

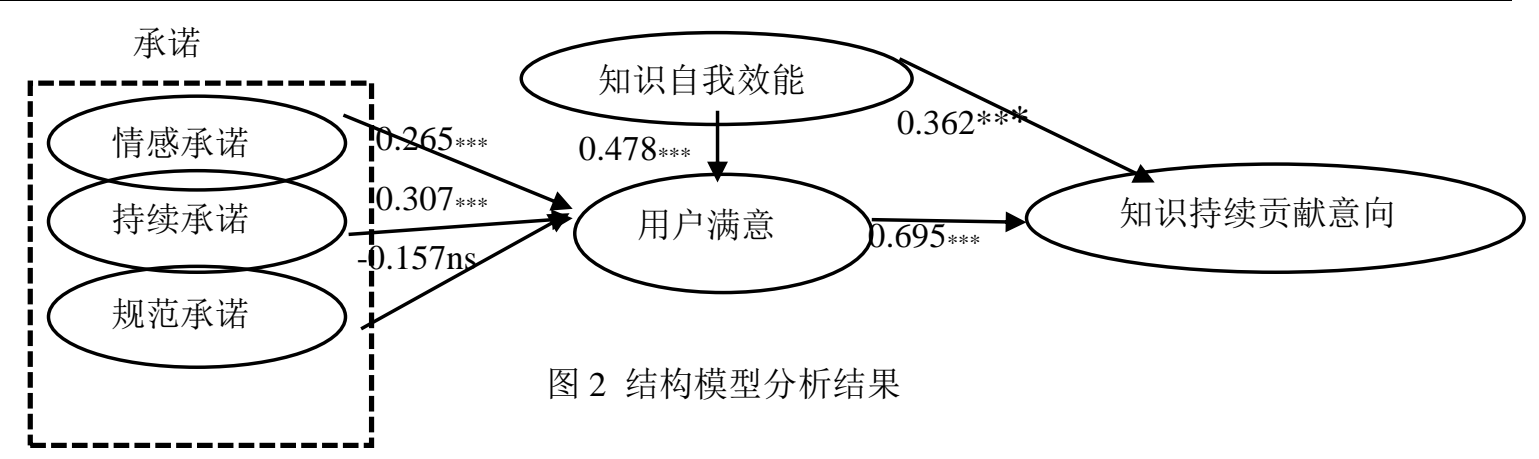




\section{6 研究发现}

表 3 是本文提出的研究模型的支持情况。

表 3 研究假设检验结果

\begin{tabular}{c|c|c}
\hline 序号 & 研究假设 & 检验结果 \\
\hline $\mathrm{H} 1$ & 情感承诺正向影响社交问答平台的用户满意度 & 支持 \\
\hline $\mathrm{H} 2$ & 持续承诺正向影响社交问答平台的用户满意度 & 支持 \\
\hline $\mathrm{H} 3$ & 规范承诺正向影响社交问答平台的用户满意度 & 不支持 \\
\hline $\mathrm{H} 4$ & 知识自我效能正向影响用户参与社交问答平台的满意度 & 支持 \\
\hline $\mathrm{H} 5$ & 知识自我效能正向影响用户参与社交问答平台的意向 & 支持 \\
\hline $\mathrm{H} 6$ & 用户满意度与其持续参与社交问答平台知识贡献意向正相关 & 支持 \\
\hline
\end{tabular}

\section{7 研究讨论}

（1）情感承诺（0.265）对用户满意度有显著的正向影响。换而言之，用户对社交问答 平台的情感承诺能够在很大程度上正向影响用户的满意度，从而促进用户持续参与社交问答 社区知识贡献行为。

（2）持续承诺（0.307）对用户满意度有显著的正向影响，且在组织承诺中是最显著的。 这一结论与本文的研究假设一致, 以知乎为代表的 UGC 内容生产方式下的社交问答平台用户 对平台的持续承诺更能影响用户的满意度，进而影响其持续知识贡献意向。

（3）规范承诺（-0.157）对用户满意度有负向影响。也就是说, 用户感知到的义务和责 任越多，其对使用社交问答平台的满意度越低，越不愿意继续参与问答社区知识贡献。

（4）知识自我效能（0.478）对用户满意度有显著的正向影响, 知识自我效能 (0.362) 对持续知识贡献意向也有显著的正向影响, 而且知识自我效能对用户满意度的路径系数大于 用户承诺的任何一个维度（情感承诺和持续承诺对用户满意度影响的路径系数分别为 0.265 和 0.307 )。

(5) 用户满意度对其持续参与社交问答平台知识贡献意愿的影响十分显著, 路径系数达 到了 $0.695 （ \mathrm{p}<0.001)$ 。换而言之, 用户对社交问答平台满意度越高, 其持续参加知识贡献的 意愿就越强。

（6）用户满意度对知识自我效能和持续知识贡献意向具有部分中介作用，知识自我效能 对知识持续贡献意向的直接效果小于知识自我效能对知识持续贡献意向的间接效果。

\section{8 研究结论}

本研究表明, 情感承诺、持续承诺和知识自我效能均对用户满意度有显著的正向影响, 且三者中知识自我效能的影响最大; 规范承诺对用户满意度没有影响; 用户满意度是知识自 我效能和知识持续贡献意愿的部分中介变量。

本文为教育部人文社科项目《珠江三角洲地区产业技术自主创新模式演化研究》(项目编 号：10YJA630080）的阶段性成果之一。

\section{References}

[1] Becker H S. Notes on the concept of commitment [J]. American journal of Sociology, 1960:32-40. 
[2] Buchanan B. Building organizational commitment: The socialization of managers in work organizations [J]. Administrative science quarterly, 1974:533-546.

[3] Mowday R T, Steers R M, Porter L W. The measurement of organizational commitment [J]. Journal of vocational behavior, 1979.14(2):224-247.

[4] Nov O, Ye C. Community photo sharing: Motivational and structural antecedents [J]. 2008. 